\title{
Effective Laparoscopic Catheter Insertion Technique for Peritoneal Dialysis Using Specially Designed Trocar: 22 Years Multi-Center Retrospective Study of 804 Patients
}

\author{
Zoran Čala1, Ivo Soldo², Zdravko Perko3, Nikola Kneževićc ${ }^{4}$, Ivica Kocman'5, Vinko Alfier6, \\ Miodrag Gudelj ${ }^{7}$, Anđelko Koprek8, Mario Kordić ${ }^{9}$, Edo Matošević10, Irena Senečić-Čala ${ }^{11}$, \\ Jasenka Kraljević ${ }^{3}$, Tadeja Pintar ${ }^{12}$
}

${ }^{1}$ Department of Surgery, General Hospital Ogulin, Ogulin, Croatia

${ }^{2}$ Department of Surgery, University Hospital Sveti Duh, Zagreb, Croatia

${ }^{3}$ Department of Surgery, University Hospital Split, Split, Croatia

${ }^{4}$ Department of Urology, University Hospital Center Zagreb, Zagreb, Croatia

${ }^{5}$ University Hospital Merkur, Zagreb, Croatia

${ }^{6}$ Department of Surgery, General Hospital Šibenik, Šibenik, Croatia

${ }^{7}$ Department of Surgery, University Hospital Rijeka, Rijeka, Croatia

${ }^{8}$ Department of Surgery, General Hospital Varaždin, Varaždin, Croatia

${ }^{9}$ Department of Urology, University Hospital Mostar, Mostar, Bosnia and Herzegovina

${ }^{10}$ Department of Surgery, General Hospital Pula, Pula, Croatia

${ }^{11}$ Department of Pediatrics, University Hospital Center Zagreb, Zagreb, Croatia

${ }^{12}$ University Medical Center Ljubljana, Ljubljana, Slovenia

Email: tadeja.pintar@kclj.si

How to cite this paper: Čala, Z., Soldo, I., Perko, Z., Knežević, N., Kocman, I., Alfier, V., Gudelj, M., Koprek, A., Kordić, M., Matošević, E., Senečić-Čala, I., Kraljević, J. and Pintar, T. (2018) Effective Laparoscopic Catheter Insertion Technique for Peritoneal Dialysis Using Specially Designed Trocar: 22 Years Multi-Center Retrospective Study of 804 Patients. Surgical Science, 9, 135-147.

https://doi.org/10.4236/ss.2018.94015

Received: December 29, 2017

Accepted: April 17, 2018

Published: April 20, 2018

\begin{abstract}
Introduction: Literature reveals several peritoneal dialysis laparoscopic catheter insertion techniques developed to improve long-term results for treatment chronic kidney failure with the technic of peritoneal dialysis. The purpose of the study is evaluation of developed and recommended minimally invasive laparoscopic technic for chronic peritoneal dialysis catheter placement using specially constructed trocar. Materials and Methods: Retrospective study included 804 patients in 10 departments of surgery. Surgical and non surgical complications related to PD catheter placement were analysed: bleeding, dialysate leak, early SSI, peritonitis, catheter tip migration, catheter obstruction, omental wrapping and visceral perforations. Available software (Microsoft $\mathrm{f}^{\oplus}$ Excel for Windows 10, MedCalc, Mariakerke, Belgium) was used for statistical analysis (presented as percentages, mean \pm SD or median). Con-
\end{abstract}


Copyright (c) 2018 by authors and Scientific Research Publishing Inc. This work is licensed under the Creative Commons Attribution International License (CC BY 4.0).

http://creativecommons.org/licenses/by/4.0/ clusions: The presented technique with specially constructed trocar is a simple and effective procedure with fewer complications comparing to literature. The advantages of this method include long rectus sheath tunnel with the deep cuff placed pre-peritoneally, the small size of the entrance into the peritoneum and accurate position and control of catheter tip in the pelvis.

\section{Keywords}

Minimally Invasive Surgical Technic, Laparoscopic Catheter Insertion,

Peritoneal Dialysis, Peritoneal Catheter, Trocar For Laparoscopic

Catheter Insertion

\section{Introduction}

Peritoneal dialysis $(\mathrm{PD})$ is an established alternative to hemodialysis for renal replacement therapy in patients with the end-stage renal disease. One of the most important prerequisites for successful peritoneal dialysis is a well-functioning catheter that permits consistent dialysis solution bidirectional flow. However, complications of the PD catheter insertion such as bleeding, dialysate leakage and malfunction, exit-site infections, peritonitis and pain may require removal of the catheter and loss of PD as dialysis modality. Literature data are positive to PD catheter irregular insertion technique being highly related to the incidence of surgical and also non-surgical complications [1] [2] [3].

Currently, several surgical techniques and modifications have been described and used for the PD catheter placement. Each method offers benefits per se, but open surgical and laparoscopic techniques are preferred in terms of safety, catheter survival and reported complications [4] [5] [6] [7] [8]. Also highly important, minimally invasive laparoscopic approach, allows direct visualization and precise catheter positioning; advanced laparoscopic surgical technics, also named additional maintaining procedures including partial omentectomy, adhesiolysis, biopsies and diagnosis and/or treatment of accompanying surgical pathologies during the same operation are mandatory in the circumstances of presented pathologies. Therefore, the number of laparoscopic peritoneal catheter placement procedures is rising around the world, as well as in Croatia, and neighboring countries [9] [10] [11] [12]. Global burden of chronic kidney disease continues to increase and consequently also the need for renal replacement therapy, clearly presented in the last literature. Due to quality hospital and outpatient medical care service outcomes of patients treated with peritoneal dialysis are better comparable to those with hemodialysis. Further growth or trends in peritoneal dialysis use is also the reflection to patient's needs, being more avtoregulative, independent and furthermore allows ordinary daily activities.

Laparoscopic PD catheters implantations using conventional laparoscopic equipment started in 1993 at the University Hospital Sveti Duh, Department of Surgery, Zagreb, Croatia. However, using the standard laparoscopic instruments, 
relatively high incidence of PD catheter insertion technic complications or technical failures was observed. To reduce the incidence of $\mathrm{PD}$ failure due to catheter placement technic based on personal data we developed several modifications of the trocar for PD catheter insertion and surgical approach in order to overcome limitations of available instruments [13]. Technically improved trocar combined to catheter placement surgical procedure conceived in 1994 is still in use today. In the last 22 years, this trocar has been distributed to surgeons and urologists in Croatia, Slovenia and Bosnia and Herzegovina with good results regarding functional and technical aspects of catheter placement [4] [14] [15] and also to promote minimally invasive surgical approach in the view to promote PD as treatment modality. The purpose of this retrospective study is to describe and evaluate designed laparoscopic technique, the trocar developed and investigate the outcomes.

\section{Methods and Patients}

1) Patients. Retrospective analysis of 804 patients with the end-stage renal disease that underwent laparoscopic PD catheter placement using specially designed trocar from September 1993 to January 2016 in eight Departments of Surgery and two Departments of Urology in Croatia, Slovenia and Bosnia and Herzegovina is presented. All operations were performed by experienced laparoscopic surgeons, previously well educated in the technic of PD catheter placement and the use of trocar. Departments with less than 25 laparoscopic insertions have not been included in this study. Postoperative follow up was performed by same surgeon and data collection.

2) Surgical procedure. Preoperative multimodal patient preparation included physical examination and education by the surgeon, nephrologist and PD nurse started a few days before surgical procedure. Preoperatively surgical procedure planning started with catheter path marking, the catheter insertion and exit site, and the cuffs position. Detailed preoperative diagnostic pull was used to exclude the presence of other abdominal pathologies to plan concurrent surgery if indicated. Routine prophylactic antibiotic use, based cefazolin or vancomycin schema was administered half an hour prior to start the procedure performed under general anesthesia. The patient was placed in the supine position; in simultaneous laparoscopic operation for other pathologies indicated, the patient was temporarily positioned for the operation and then repositioned to standard supine for the PD catheter insertion at the end of the procedure. Standard laparoscopic equipment was used along with specially designed trocar dedicated for PD catheter insertion. $12 \mathrm{mmHg}$ pneumoperitoneum was obtained by $\mathrm{CO}_{2}$ insufflation using the Veress needle or the Hasson trocar; 5 or $10 \mathrm{~mm}$ trocar was inserted through the same supraumbilical incision and 5 or $10 \mathrm{~mm}, 30^{\circ}$ or $0^{\circ}$ laparoscope was inserted for surgical exploration to peritoneal cavity. Lysis of interfering adhesions was performed, if needed. The additional $5 \mathrm{~mm}$ trocar was positioned in the left mid-clavicular line in the left or right lower abdominal 
quadrant, due to predicted position and atraumatic grasper or other working instruments were introduced through same trocar.

The trocar, dedicated for the catheter implantation, was inserted through 12 $\mathrm{mm}$ incision, on the right or left side, lateral to the umbilicus (Figure 1(a)); the exact position of incision corresponded to the high and boy weight to the patient, patient dexterity and planned home circumstances or position for PD procedure. The reusable trocar has two essential parts, the outer (sleeve) and the inner part (trocar). The outer part is a cannula, that consists of a proximal longer and wider part $(15 \mathrm{~cm}$ long, $8 \mathrm{~mm}$ in internal diameter) that penetrates through the abdominal wall extra peritoneally and a distal shorter and narrow portion ( $3 \mathrm{~cm}$ long, $5 \mathrm{~mm}$ in internal diameter) inserted intraperitoneally. The outer cannula can be longitudinally divided into two symmetric halves (Figure $1(\mathrm{~b}))$. The inner part is the trocar that, as well, consists of a longer $(7.5 \mathrm{~mm}$ in

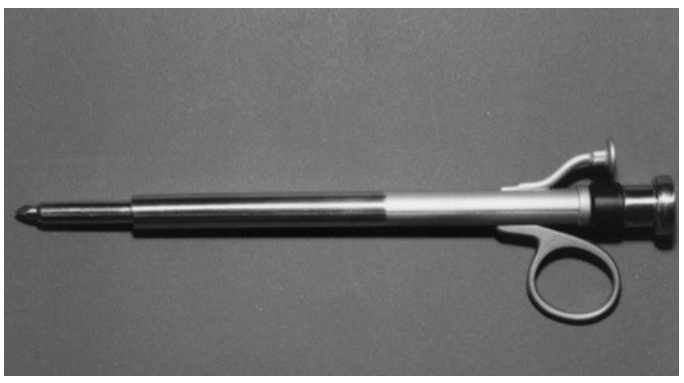

(a)

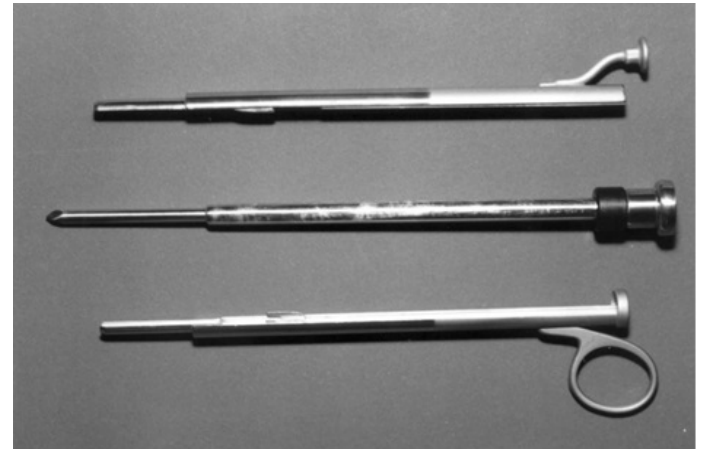

(b)

Figure 1. Trocar for laparoscopic peritoneal dialysis catheter placement (Čala \& Medaž). (a) The trocar consists of the two essential parts. The inner part consists of a longer $(7.5 \mathrm{~mm}$ in diameter) guide and a shorter outer part of consists with the pyramid-sharpened tip extending from the distal opening of the cannula (5 $\mathrm{mm}$ in diameter); (b) The outer cannula of the trocar longitudinally divided in the two symmetric halves. The outside part is a metal cannula, with a narrow portion that is introduced intra-abdominaly with short portion, inside diametter of peritoneal catether and slightly dilatates the catether path; and a larger portion that penetrates into the abdominal wall. 
diameter) and a shorter part (5 $\mathrm{mm}$ in diameter) with the pyramid-sharpened tip extending from the distal opening of the cannula. An oblique manner of trocar insertion through the abdominal wall in the craniocaudal direction forming a long canal through the body of the rectus muscle represents the first key point of the procedure; after light through of the abdominal wall the described approach significantly reduces abdominal wall trauma, incidence of bleeding, allows proper cuffs position and consequently reduces the incidence of leak and surgical site infection incidence.

The anterior rectus sheet is perforated by the sharp tip of the trocar and directed toward the pelvis within the rectus muscle and above the posterior rectus sheet. Insertion of the trocar was laparoscopically controlled with the sharp tip of the trocar clearly visible through the parietal peritoneum and the posterior rectus sheet. The abdominal organs and the inferior epigastric vessels under the peritoneum need to be clearly visualized. The peritoneum is perforated by the narrow part of the trocar in the arcuate line after achieving adequate length $(\sim 5$ $\mathrm{cm}$ long) of the rectus sheet tunnel, which depend on the patient's anthropometric properties. To proceede, the inner part is pulled out and the Tenckhoff catheter is introduced through the cannula. The deep cuff is stopped at the narrow part of the trocar i.e. to prevented significant loss of the pneumoperitoneum. Both straight and coiled double-cuff catheters are used. The coiled tip catheter was inserted through the narrow part of the trocar by rotating it inside the cannula or by using the internal straightening guidewire. The precise position of the catheter tip inside the pelvis was achieved by using the forceps through the second $5 \mathrm{~mm}$ trocar or using the guide wire under laparoscopic supervision. The cannula is then divided longitudinally into two halves must be pulled out separately. The clear visualization and control of the deep cuff position is achieved by gentle pulling motion until the posterior rectus sheet. The narrow part of the trocar ensured the minimal size of the peritoneal defect at the catheter entering site and preperitoneal position of the deep cuff. When optimally inserted, the opening in the peritoneum at the catheter inlet site was completely occupied by the catheter; proper position allows optimal peritonisation of the peritoneal catheter insertion site and those prevents leak and subsequent infection.

The abdominal cavity and the catheter position is examined between each of the preceding steps. The completion of the catheter implantation procedure requests a catheter patency; half a liter of dialysate or physiologic solution inflow and outflow catheter function and no leakage. The proximal part of the catheter with the subcutaneous cuff is pulled through the subcutaneous tunnel to the lateral exit site using a Redon needle. The subcutaneous cuff is positioned at least 3 $\mathrm{cm}$ away from the exit site to prevent possible cuff extrusion and reduce tunnel infection. No suture fixation is performed at the exit site that is covered with the non-occlusive dressing. The laparoscope is removed, and the pneumoperitoneum deflated. The fascia of the laparoscopic port sites is obliged to close with a resorbable suture to prevent incision hernia formation and promote early PD. 
Skin incisions were sutured with resorbable skin interrupted sutures.

Peritoneal dialysis was generally postponed for at least 2 weeks after the PD catheter insertion to allow complete wound healing, except in cases when the urgent PD was mandatory.

Statistical analysis. Intraoperative and early postoperative results of PD catheter implantation technic were retrospectively analyzed using patients' medical records transformed into electronic database. Follow-up time was defined as from the day of laparoscopic placement of PD catheter to day 30 postoperatively. Data analysis was performed by employing Microsoft ${ }^{\circledR}$ Excel for Windows 10 and commercially available software (MedCalc, Mariakerke, Belgium) and is presented as percentages, mean \pm SD or median.

\section{Results}

A total of 804 patients underwent laparoscopic PD catheter placement; the number of the procedures per surgeon ranged between 27 and 216 . Intraoperative and 30 days postoperative course were analyzed. Patients' ages ranged between 18 and 69 years, with a median age of 52 years. Mean operative time (skin to skin) was 32 minutes (range, 15 - 72 minutes).

Previous abdominal operations were not a contraindication for laparoscopic $\mathrm{PD}$ catheter implantation. In patients with prior abdominal surgery the open technique trocar placement was used for the access to the peritoneal cavity. No complications associated with the additional operations were recorded. Laparoscopic PD catheter implantation was performed in four patients with a vascular graft implanted more than 4 months before surgery. Advanced laparoscopic abdominal operations were performed before the PD catheter implantation in $140(17 \%)$ patients (Table 1). Before the catheter implantation, 21 (2.6\%) inguinal and $4(0.4 \%)$ umbilical hernias were repaired and concomitant hernia repair was performed for twelve (1.4\%) inguinal and two $(0.2 \%)$ umbilical hernias among that Lichtenstein procedure in 7 cases, while endoscopic total extraperitoneal procedure (TEP) in 5 cases. Two umbilical hernias were repaired using the open procedure, after removal the trocar and used for access to abdominal cavity. The peritoneum was closed in a water-tight manner in all cases. After the introduction of PD, inguinal hernia appeared in 29 (3.6\%) and umbilical hernias in $2(0.2 \%)$ patients who underwent elective surgery for hernia repair afterward. Twenty (2.4\%) laparoscopic cholecystectomies were performed for cholelithiasis in symptomatic patients. Three $(0.3 \%)$ patients were diagnosed with tumors during the laparoscopic exploration prior to catheter implantation and required concurrent salpingectomy, biopsy for carcinosis or tumor excision. Four (0.5\%) endoscopic nephrectomies and one cardiac operation, in cooperation with cardiac surgeons, were performed in one department of urology under the same general anesthesia. Detailed hemostasis was mandatory and checked after dialysate liqiud test. The abdominal drain was left in place for 24 hours in patients with concomitant abdominal operations. Adhesiolysis was necessary before the 
Table 1. Concomitant surgical procedures performed before, during and after laparoscopic peritoneal dialysis catheter (LPDC) insertion.

\begin{tabular}{cccc}
\hline & \multicolumn{3}{c}{ LPDC $(\boldsymbol{n}=\mathbf{8 0 4})$} \\
\cline { 2 - 4 } & Before LPDC & During LPDC & After LPDC \\
\cline { 2 - 4 } & $\mathbf{n}(\%)$ & $\mathbf{n}(\%)$ & $\mathbf{n}(\%)$ \\
\hline Surgical procedures & $21(2.6 \%)$ & $12(1.4 \%)$ & $29(3.6 \%)$ \\
Inguinal hernia repair & $4(0.5 \%)$ & $2(0.2 \%)$ & $2(0.2 \%)$ \\
Umbilical hernia repair & & $31(3.8 \%)$ & \\
Lysis of adhesions & & $6(0.7 \%)$ & \\
Omentectomy & & $3(0.3 \%)$ & \\
Omentopexy & & $2(0.2 \%)$ & \\
Suture tip fixation & & $20(2.4 \%)$ & \\
Cholecystectomy & & $3(0.3 \%)$ & \\
Salpingectomy & & $4(0.5 \%)$ & \\
Nephrectomy & & $1(0.1 \%)$ & \\
Cardiac surgery & &
\end{tabular}

catheter insertion in $31(3.8 \%)$ patients, $26(84 \%)$ had prior abdominal surgery and partial omentectomy performed in $6(0.7 \%)$ and omentopexy in $3(0.3 \%)$ cases. Catheter suture fixation was performed twice $(0.2 \%)$.

The incidence of major surgical complications is listed in Table 2. No intraoperative deaths were recorded. Bleeding complications occurred in 46 (5.7\%) patients. Inferior epigastric vessels injury resulted in abdominal wall bleeding in $1 \%$ at the time of catheter implantation and in $1.7 \%$ of patients after the procedure. Wound revision, ligation, and electrocoagulation was performed in the case of bleeding; abdomial bleeding after PD catheter implantation occurred in $21(2.6 \%)$ cases, among that laparoscopic revision and hemostasis in 10 cases and the remaining stopped spontaneously with no need to blood transfusions. Self limitation of bleeding was observed day 2 postoperatively and mostly presented as initially bloody dyalisate fluid to minimal number of RBC, presented in dialysate laboratory analysis. To reduce the possible catheter clot formation small volume abdominal cavity lavage was performed. In 2 cases discrete bleeding was presented from liver after concomitant laparoscopic cholecystectomy. Early exit site infection occurred in $28(3.4 \%)$ patients average day 4 postoperatively. All patients received standard surgical wound treatment augmented with oral antibiotics. Topic antibiotic application with mupirocin ointment topical was performed in $3 / 28(10.71 \%)$ patients due to low response to wound care and systemic antibiotic application and that represented efficient treatment approach. Swab identification in all patients was positive to Staphylococcus aureus and Streptococcus pyogenes. Slight skin edema was present day 1 postoperative in all 3 patients and other local infection signs absent up to day 4 postoperatively. No identification of skin microbiota was performed prior catheter placement. Peritonitis occurred in early postoperative period (30 days) in 35 (4.3\%) 
Table 2. Complications of peritoneal dialysis catheter insertion.

\begin{tabular}{cc}
\hline & Number of patients $(\boldsymbol{n}=\mathbf{8 0 4})$ \\
\cline { 2 - 2 } Intra-operative complications & $\mathrm{n}(\%)$ \\
\hline Abdominal wall bleeding & $8(1 \%)$ \\
Abdominal cavity bleeding & $3(0.3 \%)$ \\
Visceral perforation & $2(0.2 \%)$ \\
\hline Post-operative complications & \\
\hline Abdominal wall bleeding & $14(0.7 \%)$ \\
Abdominal cavity bleeding & $21(2.6 \%)$ \\
Dialysate leak & $23(2.8 \%)$ \\
Exit site infection & $28(3.5 \%)$ \\
Peritonitis & $35(4.3 \%)$ \\
Abdominal pain & $7(0.9 \%)$ \\
Catheter tip migration & $18(2.2 \%)$ \\
Blood clot/Fibrin plug obstruction & $21(2.6 \%)$ \\
Omental wrapping & $6(0.7 \%)$ \\
Death & $1(0.1 \%)$ \\
\hline
\end{tabular}

patients and treated combined with intravenous and intraperitoneal antibiotics. In 5 refractory cases catheter was removed due to persistent positive dialysate swab and systemic infection. Analysis revailed positive cultures to Escherichia coli and Klebsiella pneumoniae, intraoperatively no acsites or signs of peritonitis were observed in all patients and also no liver pathology or vascular grafts present. In all patients skin insertion site (SSI) was negative.

Dialysate fluid leakage occurred in $23(2.8 \%)$ in the first 30 days after the catheter insertion and treated with low volume PD or temporary transfer to hemodialysis for a few weeks (average 4 to 6 ). There were two (0.2\%) visceral perforations during catheter implantation. In one patient, the gastric perforation with Veress needle required open surgical approach and the second case was small intestinal perforation after intestinal loop adhesiolysis with consequent development of diffuse peritonitis three days after. The patient suffered from several comorbidities and despite timely surgical and intensive therapy, overall clinical deterioration progressed to death. Peritoneal dialysis catheter malfunction occurred in $5.6 \%$ of patients. Failure was due to catheter tip migration in $2.2 \%$, blood clot or fibrin plug obstruction in $2.6 \%$, and omental wrapping in $0.7 \%$. It was treated by non-operative measures such as flushing and thrombolytics which have been proven effective. Catheter malfunction due to catheter tip migration or constipation was treated with fluoroscopic wire manipulation. Patients with malfunctioning peritoneal dialysis catheters due to omental wrapping underwent laparoscopic exploration and restoration of omentum and catheter tip. Seven patients suffered abdominal pain after infusion of dialysate fluid, however the pain resolved with time and there was no need for repositioning or 
removal of the catheter. In all of these cases, a catheter with straight tip was used.

\section{Discussion}

Peritoneal dialysis is a treatment modality used worldwide for the treatment of end-stage renal disease. The PD catheter function and the incidence of the catheter-related complications strongly depend on the technique of implantation. However, in the past few decades, the controversy persists about the best insertion technique. Considering all the advantages of minimally invasive approach, laparoscopic PD catheter is the most frequently used method today and needed to become the preferred approach [10] [11] [12] [13] due to short time of full PD establishment, low rate of early complications, patients independence, working ability and also better cosmetic effect. Due to available data, expanded use of laparoscopic implantation of $\mathrm{PD}$ catheters is due to its advantages regarding better visualization of the peritoneal cavity and more precise positioning of the catheter when compared to other implantation modalities [14]. However, publications report many shortcomings of the standard laparoscopic equipment when used for PD catheter implantation with different described laparoscopic techniques for the PD catheter placement [15] [16].

The distinctive feature of described developed method is the usage of standard laparoscopic equipment with the addition of dedicated reusable trocar for catheter placement requests only minimal additional cost. Statistical data presents a low rate of technical failures and complications when compared to similar studies [17] [18] [19]. Moreover, this multi-center experience is simply and completely reproducible [13].

Our data strongly support the conclusion based on statistical data that laparoscopic peritoneal catheter implantation using our specially constructed reusable trocar is a safe, short lasting, efficient and feasible alternative to other laparoscopic techniques in the management of the patients requiring peritoneal dialysis. Visualisation of abdominal cavity, prompt hemostasis, catether tip and internal insertion point inspectation are important to reduce also the full restoration of PD and quality of life. In addition, laparoscopy is useful for the diagnosis and treatment of other pathologies, also highly important in the clinical circumstances of previously no clinical signs presented. We can conclude also, that laparoscopic cholecystectomy, nephrectomy, hernia repair and cardiac operations can be successfully performed in the same general anesthesia [20] [21].

In our series, combined operations concomitant with PD catheter placement were also performed. Seventy patients had an inguinal or an umbilical hernia and underwent hernia repair before, simultaneously or after the PD catheter insertion. Inguinal hernias were treated using the open Lichtenstein procedure or endoscopic total extraperitoneal procedure (TEP) that is preferred technique for hernia repair in PD patients due to preserved peritoneal integrity [21]. Umbilical hernias were repaired using the open method with direct suturing or Mayo technic. Three patients underwent salpingectomy and tumor excision prior to 
catheter implantation due to intraoperative finding i.e. laparoscopic exploration. Twenty laparoscopic cholecystectomies were performed for cholelithiasis in asymptomatic patients. In patients with acute cholecystitis and candidates for laparoscopic PD insertion laparoscopic cholecystectomy was performed and after a disease free interval of three weeks the procedure finalised. Four endoscopic nephrectomies and one cardiac operation were also performed and no additional surgical and non surgical complication observed related to concomitant procedures.

Third and important observation to our study is that laparoscopic method is particularly useful in patients with previous abdominal operations. In this setting, adhesiolysis is safely performed to prevent nor catheter malfunction to unssufficient PD due to reduced exposure to parietal and visceral peritoneum in the case of multiple adhesions. Importantly, the situation allows peritoneal dialysis also in patients who would otherwise require hemodialysis, as we did in 27 cases. The primary causes of catheter dysfunction, as reported in review publications and PD guidelines, are compartmentalization from adhesions, catheter tip migration and omental wrapping or entrapment [22]. Our technique of laparoscopic PD catheter placement directly addresses these issues by allowing long rectus sheath tunneling along with adhesiolysis and omentopexy [23] [24]. However, only adhesions that may obstruct catheter function should be treated. Adhesions in the upper part of the abdomen usually do not interfere with dialysate drainage and therefore does not mandate surgical management. Moreover, unnecessary adhesiolysis may increase the risk of postoperative bleeding [25] and intestinal trauma.

Although several publications recommend routine omentectomy or omentopexy, we found that unnecessary. These procedures may prolong operation, and could result in complications such as postoperative bleeding, catheter obstruction, and internal herniation [24] [25]. Omentopexy should be done selectively in patients having extremely long omentum to reduce the incidence of omental wrapping and dialysat compartmenisation. In our study, the omentopexy was performed in three cases with the additional two cases of catheter suture fixation.

Special care should be taken to avoid visceral injuries during coagulation and manipulation with the instruments. Damage to the small or large bowel may not be visible during the operation with clinical and laboratory findings presentation up to 3 - 6 days later. Although, this complication is rare $(0 \%-2 \%)$ clinical situation after laparoscopic PD catheter insertion, we reported two visceral perforations in our study. The gastric perforation was due to the Veress needle insertion and the small bowel injury occurred during adhesiolysis in low BMI patient after massive weight loss and abdominal wall distension. Recent studies also propose, that the use of peritoneal dialysis may offer advantages in patients with the intra-abdominal foreign body, such as vascular grafts, due to better hemodynamic control and avoidance of anticoagulation, also supported by our study. In our 
study, with respect of graft positioning time, one patient with vascular graft underwent laparoscopic PD catheter insertion without any complication. Free interval in between two procedures is highly recommended, mostly more than 4 months allows complication free procedures, also VGI that is an absolute contraindication for PD and PD catheter placement.

In 0 to $12.8 \%$ of patients after laparoscopic insertion of $\mathrm{PD}$ catheter dialysate fluid leakage represents delay in full PD, also well documented to published data [22]. Besides, local environment in dialysate leakage negative circumstances to SSI incidence, reduces QL and additional economical costs. The reported low rate of dialysate leakage $(2.8 \%)$ in our study is related to surgical technique, the features of our specially designed trocar and preoperative multimodal patient preparation. The trocar ensured the minimal size of the peritoneal defect at the insertion site, long rectus sheath tunnel and the deep cuff placed preperitoneally, inside the rectus sheath all above the positive consequences of dilatation approach of abdominal wall structures with minimal trauma and improved conformance to wall structures to implemented catheter. Importantly, peritoneal defect has close the same the catheter diameter that allows also rapid peritonisation and reduces leak. Using dedicated reusable trocar for laparoscopic catheter implantation keeps the catheter oriented toward the pelvis and importantly reduces the number of catheter tip migration due to prolonged rectus muscle sheat. Therefore, the need for suture fixation of the catheter is eliminated and to well described technical unnecessary procedure that is highly supported with the literature data that suture fixation of the catheter may cause an internal hernia or formation of adhesion [9] [17].

Anthropometric parameters of the patients were evaluated only in few centers included in the study and importantly, mostly in the limited period and those represent a limitation of the study and interpretation. Metabolic and nutritional adaptations in patients with CKD with clinical presentation of skeletal muscle mass and function deterioration importantly contribute to surgical risk factors calculation. In limited number of patients data obtained with BIA showed important correlation between duration of $\mathrm{CKD}$ and time of catheter placement, elevated PA (phase angle), reduced lean body mass, surgical and non-surgical postoperative complications and efficient PD establishment. Besides that, not uniform dietary counseling was used in all centers that also represents the limitation in interpretation of the results. The age of CKD patient was not the purpose of observation but also represents an important variable in risk factor stratification. Risk factor calculation due to oxidative stress in CKD might be interpreted with PA and preoperative BIA measurement results interpretation and correction of PA with treatment modalities might represent an efficient approach to reduce surgical risks. Limited data are positive for the influence of increased PA and the need of catheter removal in our study, but as the number of patients is low no evidence based conclusion might be possible. Otherwise, in multimodal patient preparation for surgery, also in patients with ESRD, anthro- 
pometric, BIA and metabolic data represent an important working field to reduce surgical and non surgical risk factors reduction.

\section{Conclusion}

The presented technique of laparoscopic PD catheter insertion with a specially constructed reusable trocar is simple to use and requires no additional training. It requires standard laparoscopic equipment besides specially constructed reusable trocar that itself poses only minimal additional cost. The procedure is also compliant with consensus guidelines for best-demonstrated practices in peritoneal access placement including long rectus sheath tunnel in craniocaudal oblique direction, toward the pelvis, with preperitoneal deep cuff. It offers excellent visualization of all procedure steps which is especially important for precise control of the peritoneal surface during the trocar insertion and positioning and besides gives optimal cosmetic effect. Therefore, this laparoscopic procedure for $\mathrm{PD}$ catheter insertion is a safe and effective procedure with low risks of morbidity and mortality. However, optimal long-term catheter function and PD depends on the effective multi-disciplinary program, ongoing re-examination and cooperation between nephrologists and surgeons.

\section{References}

[1] Čala, Z., Velnić, D., Varlaj, V., Janković, N. and Čala, S. (1994) Placement of a Catheter for Peritoneal Dialysis Using Videolaparoscopy. Surgical Endoscopy, 8, 1010.

[2] Tsimoyiannis, E.C., Siakas, P., Glantzounis, G., et al. (2000) Laparoscopic Placement of the Tenckhoff Catheter for Peritoneal Dialysis. Surgical Laparoscopy Endoscopy \& Percutaneous Techniques, 10, 218-221. https://doi.org/10.1097/00129689-200008000-00007

[3] Crabtree, J.H. (2006) Selected Best Demonstrated Practices in Peritoneal Dialysis Access. Kidney International Supplements, No. 103, S27-S37. https://doi.org/10.1038/si.ki.5001913

[4] Maheshwari, P.N., Heda, R.S., Oswal, T.A., et al. (2014) Laparoscopy-Assisted Continuous Ambulatory Peritoneal Dialysis Catheter Placement Using Amplatz dilators: A New Technique with Results. Urology, 84, 1521-1524.

https://doi.org/10.1016/j.urology.2014.08.027

[5] Crabtree, J.H. and Burchette, R.J. (2009) Effective Use of Laparoscopy for Long-Term Peritoneal Dialysis Access. American Journal of Surgery, 198, 135-134. https://doi.org/10.1016/j.amjsurg.2008.10.019

[6] Voss, D., Hawkins, S., Poole, G., et al. (2012) Radiological versus Surgical Implantation of First Catheter for Peritoneal Dialysis: A Randomized Non-Inferiority Trial. Nephrology Dialysis Transplantation, 27, 4196-4204. https://doi.org/10.1093/ndt/gfs305

[7] Markic, D., Vujičić, B., Ivanovski, M., et al. (2015) Peritoneal Dialysis Catheter Placement Using an Ultrasound-Guided Transversus Abdominis Plane Block. Blood Purification, 39, 274-280. https://doi.org/10.1159/000381005

[8] Crabtree, J.H. (2014) SAGES Guidelines for Laparoscopic Peritoneal Dialysis Access Surgery. Surgical Endoscopy, 28, 3013-3015.

https://doi.org/10.1007/s00464-014-3812-3 
[9] Haggerty, S., Roth, S., Walsh, D., et al. (2014) Guidelines for Laparoscopic Peritoneal Dialysis Access Surgery. Surgical Endoscopy, 28, 3016-3045. https://doi.org/10.1007/s00464-014-3851-9

[10] Ash, S. (2005) Laparoscopy for PD Catheter Placement: Advantages and Disadvantages versus Peritoneoscopy. Peritoneal Dialysis International, 25, 541-543.

[11] Tenckhoff, H. and Schechter, H. (1968) A Bacteriologically Safe Peritoneal Access Device. Transactions-American Society for Artificial Internal Organs, 14, 181-187.

[12] Čala, Z. (2000) Trocar for Laparoscopic Placement of Peritoneal Dialysis Catheter. Surgical Endoscopy, 14, 308-309. https://doi.org/10.1007/BF03380116

[13] Čala, Z., Velnić, D., Varlaj-Knobloch, V., Jankovič, N., Čala, S. and Perko, Z. (1997) Placement of the Peritoneal Dialysis Catheter Using Videolaparoscopy. Croatian Journal of Gastroenterology and Hepatology, 6, 7-11.

[14] Čala, Z., Mimica, Ž., Ljutić, D., Janković, N., Varlaj, V. and Čala, S. (2000) Laparoscopic Placement of the Peritoneal Dialysis Catheter Using a Specially Designed Trocar: A Review of 84 Patients. Dialysis \& Transplantation, 29, 722-727.

[15] Crabtree, J.H. and Burchette, R.J. (2006) Prospective Comparison of Downward and Lateral Dialysis Catheter Tunnel-Tract and Exit-Site Directions. Peritoneal Dialysis International, 26, 677-683.

[16] Gokal, R., Alexander, S., Ash, S., et al. (1998) Peritoneal Catheters and Exit-Site Practices toward Optimum Peritoneal Access: 1988 Update. Peritoneal Dialysis International, 18, 11-33.

[17] Figueiredo, A., Goh, B.L., Jenkins, S., et al. (2010) Clinical Practice Guidelines for Peritoneal Access. Peritoneal Dialysis International, 30, 424-429. https://doi.org/10.3747/pdi.2010.00087

[18] Crabtree, J.H. (2009) The Use of the Laparoscope for Dialysis Catheter Implantation: Valuable Carry-On or Excess Baggage? Peritoneal Dialysis International, 29, 394-406.

[19] Barone, G.W., Lightfoot, M.L. and Ketel, B.L. (2002) Technique for Laparoscopy-Assisted Complicated Peritoneal Catheter Placement. Journal of Laparoendoscopic \& Advanced Surgical Techniques, 12, 53-55. https://doi.org/10.1089/109264202753486939

[20] Crabtree, J.H. (2006) Hernia Repair without Delay in Initiating or Continuing Peritoneal Dialysis. Peritoneal Dialysis International, 26, 178-182.

[21] Čala, Z., Velnić, D., Perko, Z., Varlaj-Knobloch, V. and Čala, S. (1997) Insertion of Peritoneal Dialysis Catheter in Combination with Laparoscopic Operations. Surgical Endoscopy, 11, 593.

[22] Tzamaloukas, A.H., Gibel, L.J., Eisenberg, B., et al. (1990) Early and Late Peritoneal Dialysate Leaks in Patient on CAPD. Advances in Peritoneal Dialysis, 6, 64-71.

[23] Attaluri, V., Lebeis, C., Brethauer, S. and Rosenblatt, S. (2010) Advanced Laparoscopic Techniques Significantly Improve Function of Peritoneal Dialysis Catheters. Journal of the American College of Surgeons, 211, 699-704. https://doi.org/10.1016/j.jamcollsurg.2010.08.010

[24] Crabtree, J.H. and Fishman, A. (2005) Laparoscopic Method for Optimal Peritoneal Dialysis Access. The American Surgeon, 71, 135-143.

[25] Blake, P.G., Sloan, J.A., McMurray, S., Jain, A.K. and Matthews, S. (2014) A Multicentre Survey of Why and How Tidal Peritoneal Dialysis (TPD) Is Being Used. Peritoneal Dialysis International, 34, 458-460.

https://doi.org/10.3747/pdi.2013.00314 